\title{
Lionel Groulx : son mythe et ses mythes
}

\section{Georges-Émile Giguère}

Volume 45, 1978

URI : https://id.erudit.org/iderudit/1007122ar

DOI : https://doi.org/10.7202/1007122ar

Aller au sommaire du numéro

Éditeur(s)

Les Éditions Historia Ecclesiæ Catholicæ Canadensis Inc.

ISSN

0318-6172 (imprimé)

1927-7067 (numérique)

Découvrir la revue

Citer cet article

Giguère, G.-É. (1978). Lionel Groulx : son mythe et ses mythes. Sessions d'étude -

Société canadienne d'histoire de l'Église catholique, 45, 19-39.

https://doi.org/10.7202/1007122ar

Tous droits réservés @ Les Éditions Historia Ecclesiæ Catholicæ Canadensis Inc., 1978
Ce document est protégé par la loi sur le droit d'auteur. L'utilisation des services d’Érudit (y compris la reproduction) est assujettie à sa politique d'utilisation que vous pouvez consulter en ligne.

https://apropos.erudit.org/fr/usagers/politique-dutilisation/ 


\section{Lionel Groulx : son mythe et ses mythes}

L'année 1978 qui s'achève aura donné lieu à diverses manifestations toutes destinées à signaler le centenaire de naissance du chanoine Lionel Groulx. En évoquant ainsi le souvenir de notre historien national, que demeure toujours notre abbé Groulx, on a voulu simplement retrouver, au contact de ce grand Québécois, l'inspiration même qui lui a permis de rendre de si grands services à ses concitoyens. C'était, n'est-ce pas, agir en toute vraisemblance, selon une coutume immémoriale que de retourner dans le passé pour en revenir chargé de leçons profitables pour le bénéfice du présent comme pour l'avantage de l'avenir. Quel autre geste posent les hommes d'affaires ou les chefs politiques qui veulent évaluer leur année, leur mandat et préparer leur bilan, leurs prévisions à venir?

Pourtant, à l'encontre de cette pratique, certains esprits chagrins ont voulu y voir plutôt une invitation lancée aux Québécois pour qu'ils aillent collectivement planter leur tente dans le temps passé, pour aller s'installer et vivre un siècle ou un demi siècle en arrière. Voilà un bien drôle de raisonnement qui a tendance à vouloir se répandre ${ }^{1}$.

À cause du titre donné à trois de ses volumes, Lionel Groulx est devenu le symbole de «NOTRE MAÎTRE LE PASSÉ ». Pour le monde ordinaire, comme pour Lionel Groulx lui-même ou encore pour André Beaumier à qui il a emprunté cette formule, "NOTRE MAÎTRE LE PASSÉ » c'est une vérité de la Palisse, c'est un slogan par lequel on admet sa propre dépendance à l'égard du passé, à l'endroit des ancêtres et du pays qui nous a vus naître. Ce qui ne veut pas dire qu'on se soumet, ni qu'on se résigne même aux con-

1 La Presse du 23 octobre 1978 reproduisait le texte que voici : "Le centenaire Lionel Groulx n'est qu'une vulgaire tentative de recyclage par nos péquistes, nos marchands de livres et nos have been nationaleux de Groulx, ecclésiastique porteur d'idéologie, autoritaire, agriculturiste, admirateur du Duce et de Salazar, raciste à l'endroit des Amérindiens ». 
séquences néfastes. Regarder le passé, c'est découvrir, en outre du chemin parcouru, la façon dont la marche en avant s'est faite, les retards qu'elle a subis, les causes et conditions du progrès comme celles des retards.

Bien loin d'être un objectif à poursuivre à tout prix, comme le prétendent encore ces mêmes gens, notre passé demeure la source de notre avenir. Simple mise au point, me direz-vous, mais qu'il fallait faire pour écarter dès le début toute erreur de perspective et d'interprétation. Vaine subtilité peut-être pour les lecteurs d'une biographie populaire, pour le public en général, pour l'homme de la rue ou pour $\mathbf{M}$. et $\mathbf{M}^{\mathrm{mc}}$ Tout-le-monde, à qui il suffit de connaître rapidement et facilement Lionel Groulx. Mais ces problèmes de spécialistes éviteront même à tous ces gens de se faire attraper par la bande. Voilà pourquoi il faut les aborder en leur rappelant que Groulx demeure un sujet complexe et extrêmement riche à étudier, d'abord à cause de l'immense bibliographie qui le concerne, et de la diversité et de la durée de son activité.

Personnage aux mille facettes, Lionel Groulx est d'autant plus difficile à atteindre et à comprendre. Cependant nous croyons le résumer correctement en affirmant que, tout historien qu'il était, Groulx fut davantage tourné vers l'avenir que vers le passé. Ne criait-il pas à Québec en 1937 à tous les pessimistes, les défaitistes, les déprimés, les profiteurs et les inactifs : «Vous êtes la dernière génération des morts », pour ensuite ajouter en se tournant vers les courageux, les vaillants, les optimistes : "Nous sommes la première génération des vivants. Notre État français nous l'aurons!»

Reconnaître qu'on est tributaire du passé tant dans notre être que dans nos façons de penser et d'agir, n'a rien que de très normal. Bien loin d'être un crime, c'est le moyen le plus sûr de ne pas demeurer prisonnier, ni d'être la victime inconsciente et impuissante de ce passé, si jamais il y a lieu. C'est même la manière tout indiquée de s'en sortir, de retrouver ses énergies, de se donner des motivations. Au contraire l'erreur consiste à ignorer le passé, son passé, à ne pas vouloir connaître même les antécédents théoriques, ou mieux encore vécus, des idéologies qu'on prétend soutenir.

En histoire, la première opération consiste à établir les faits et à les coordonner. Nécessaire et indispensable, cet exercice demeure ordinairement facile. Mais il n'est pas le plus important à côté des idéologies à démêler. Un tel triage est une entreprise plus 
compliquée et combien plus obligatoire, sans aucun doute, à cause des conséquences qui en découlent. Or on sait que Groulx fut à lui seul toute une école de pensée, louée et parfois exaltée par les uns, critiquée et tantôt vertement blâmée par les autres. Qu'en est-il au juste? Il faut assurément être bien conscient de ses propres idéologies avant d'étudier, de juger, d'accepter ou de condamner celles des autres. Qu'on ait ou non partagé les idées et les idéaux de Groulx, on doit reconnaitre qu'il y avait chez lui beaucoup de grandeur et d'intelligence, qu'il fut un énorme travailleur et qu'il a exercé un très large rayonnement.

Quant aux attaques qu'il a subies de son vivant, grâce à Dieu, L.G. a su se défendre avec autant de vigueur que de grandeur d'âme. Cependant, peut-on se demander, se pourrait-il que les plus mesquines attaques, comme les plus perfides, soient venues depuis sa mort? Elles se situent à la fois au niveau des faits et au niveau des interprétations. Et à en juger par les carrefours d'information occupés par ses dénonciateurs, on peut déduire qu'ils se sont infiltrés partout. Et tout cela nous conduit à poser deux questions majeures, concernant soit la louange qui aurait pu atteindre jusqu'au mythe soit la critique qui aurait pu lui prêter certains mythes qu'elle dénonce. Mais, attention! Pour éviter de nous en enfarger dans les mots, nous formulerons comme suit nos questions :

A - Lionel Groulx fut-il bien le grand homme qu'on nous a toujours dit?

B - Lionel Groulx fut-il le grand méchant homme qu'on veut parfois nous faire croire?

\section{A - LIONEL GROULX FUT-IL LE GRAND HOMME QU'ON NOUS A DIT?}

Depuis l'époque où les premiers historiens ont écrit, c.a.d. depuis Hérodote et Thucydide, un phénomène s'est plusieurs fois reproduit au sein des écoles historiques. Ce phénomène qui s'appelle en français "personnalité incorporante» et en anglais "corporate personality » consiste à donner à une collectivité le nom d'une personne généralement reconnue comme telle. Le procédé est attribuable au côté mystérieux du personnage soit à cause de son éloignement dans le temps, soit encore à cause de la polyvalence de son être ou de son œuvre. Autrement dit, pour expliquer l'inex- 
plicable, on en fait un être multiple, un collectif. Le procédé de "personnalité incorporante " a ainsi recouvert d'abord Adam, ensuite le poète Homère, puis le Christ, enfin Shakespeare et finalement Napoléon.

Cependant qu'on se rassure, tel n'a pas encore été le cas de Lionel Groulx, puisque onze ans après sa mort, de nombreux témoins et contemporains lui survivent toujours. Bien plus il a tellement écrit, il a tellement répondu aux questions et objections, il a été si présent à son époque et à son milieu, que les témoignages sur sa personnalité ou sur son œuvre n'offrent que peu d'ambiguïté à qui sait les consulter et les lire. Cependant si l'on veut éviter que Groulx devienne un de ces êtres collectifs, la première exigence de méthode consiste à chercher la véritable teneur de sa pensée dans les ouvres même de Groulx et non ailleurs. En contrepartie il $\mathrm{y}$ a trois pratiques à éviter : 1- confondre Groulx avec certains de ses contemporains, comme Henri Bourassa ou Thomas Chapais, 2- scruter de préférence les paroles et écrits de ses disciples ${ }^{2}$, 3- aller plutôt aux commentaires et aux critiques faites sur Groulx et son œuvre. De telle sorte que Groulx ne soit pas Légion et qu'on ne dise pas davantage que Son nom est personne.

L'existence de Lionel Groulx qui s'est terminée dans sa $90^{\mathbf{e}}$ année, a été des plus actives jusqu'à la veille de sa mort le 23 mai 1967. Son œuvre autant que sa vie a été polyvalente tout comme le siècle qui s'est déroulé depuis 1878. Ces 90 ans, plus les dix ans qui nous séparent de sa disparition, forment non pas un siècle, mais plus exactement cinq tranches de vingt ans de vie consciente et responsable. Cinq tranches qui chacune ont été indéniablement marquées d'un progrès, d'une évolution sur la précédente. Loi élémentaire qui régit toute vie individuelle comme toute mentalité et tout milieu, autant que tout pays comme l'univers lui-même. Rien là que de très normal en histoire, comme ça devrait être normal pour toute science humaine.

Or ce siècle (1878-1978) a assisté à la naissance de la photographie, de vues fixes et de petites vues, du cinéma muet puis parlant, de l'électricité, de l'automobile, de la radio et de la télévision, de l'aviation et même des supersoniques, des transports et communications de plus en plus rapides, de la bombe atomique et

2 Maurice Tremblay, "Réflexions sur le nationalisme», in Écrits du Canada français V, 1959, pp. 10-43. 
de l'énergie nucléaire. Et que d'autres nouveautés et utilités nous laissons de côté ! Durant ce même siècle des États ont vu s'implanter chez eux la dictature ou le marxisme-léninisme comme en URSS et chez ses satellites, ou avec des variantes comme à Cuba et en Chine et chez leurs affiliés. Plus près de nous, nos propres gouvernements ne sont-ils pas devenus récemment plus conscients de leurs responsabilités sociales, comme par exemple de la politique culturelle qui chez nous n'a pas encore terminé sa naissance. Il n'y a pas tellement longtemps qu'on parle de bourses ou de subventions à la recherche, d'aide à l'édition, de bibliothèques publiques ou de politique du livre, sans oublier l'organisation des archives et la préparation d'instruments de travail. Que de chemin parcouru tant dans le monde universitaire que scientifique! Ne serait-ce que depuis l'avènement de la sociologie, des sciences politiques ou économiques, et cela va sans dire de l'évolution de la science historique, de la conception qu'on s'en est faite comme des méthodes auxquelles on a recouru. Dans tout cela il y a eu des débuts, puis des étapes marquées de progrès. Tout cela s'est produit depuis la naissance de Groulx.

Lionel Groulx, comme nous l'a rappelé opportunément Maurice Filion, a été "à la fois le témoin et l'agent d'une tranche de notre histoire marquée d'événements importants : le début de la Confédération, la résurgence de l'impérialisme britannique, le nationalisme de Bourassa, la guerre de 1914-1918 et la conscription, la crise économique de 1929, le Statut de Westminster, la guerre de 19391945, la naissance et la fin du Bloc populaire, l'ère Duplessis et la Révolution tranquille » 3 . À l'énumération faite par Filion il nous faudrait ajouter l'urbanisation et l'industrialisation qui n'ont pas épargné la province de Québec. Et Groulx est disparu au moment où allait s'ouvrir l'Exposition universelle de 1969 et où on soulignait les cent ans d'invariabilité du BNAA (AANB), invariabilité que Groulx avait favorisée tant par l'inapplication que par le non respect.

Revenir ainsi sur les étapes chronologiques et en tenir compte dans ses évaluations pour les cinq tranches qui composent ce siècle est donc une exigence élémentaire de l'historien comme du sens de l'histoire. Comment peut-on négliger ces grandes marches, ces degrés du progrès quand on veut mesurer à sa juste portée une œuvre

3 Maurice Filion, Introduction in Hommage à Lionel Groulx, Leméac, 1978. 
comme celle de Groulx qui s'est étalée sur quelque soixante-cinq ans, soit les 65 dernières de sa vie ? Est-il besoin d'insister davantage?

$\mathrm{Au}$ plan de la production, l'activité de Groulx a abouti à quelque 30 volumes, sans tenir compte du nombre de tomes et d'éditions pour certains, une quarantaine de brochures et autant de lettres-préfaces, plus de 200 articles de journaux et revues, pas moins de 240 critiques de livres, sans oublier 10 ans à la direction de L'Action française et 20 ans à la tête de la Revue d'histoire de', l'Amérique française. Cette revue trimestrielle qui publie annuellement 650 pages a atteint un total de 13,000 pages en 20 ans. Et nous ne parlons pas des nombreux inédits (le P. Benoit Lacroix mentionne plus de 1,000 manuscrits) ${ }^{4}$, des conférences, des prédications et des cours devenus plus tard des livres ou des brochures, Le tout prend place dans l'œuvre historique ou dans l'œuvre oratoire quand ce n'est pas dans les varia qui ont reçu la forme de poésies, de romans, de contes ou de controverses et qui, avec la correspondance et les coupures de journaux, ont fini par composer quelque 80 spicilèges. Voilà sans doute une appréciation toute quantitative qu'on ne peut vraisemblablement pas contester et dont la moindre partie nous rendrait tous orgueilleux.

Si nous passons du côté qualitatif, Groulx fut un homme de volonté et de devoir, d'intelligence et de jugement, un audacieux et un courageux, un optimiste et un grand espérant. Il a écrit, il a parlé. Par la plume et par la parole, il fut un irrésistible communicateur. Mais précisément pour avoir fait la navette entre l'idéologie nationaliste et le réalisme de l'histoire, il a établi des liens que certains lui reprochent. Au contraire Groulx a prétendu par là servir une histoire plus incarnée, donc plus près de la réalité quotidienne. Alternativement il s'est retrouvé dans la chaire du professeur puis sur la tribune de l'orateur. Il quittait la parole pour retrouver la plume. De la sorte son style n'est pas écrit, mais presque toujours parlé, ce qui vauit à ses écrits historiques l'accusation de n'avoir pas toujours résisté à l'emportement de l'art oratoire. Guy Frégault considère pour sa part que Groulx a trop souvent quitté l'histoire pour accomplir d'autres tâches. D'autres trouvent que répondant et s'adaptant aux nécessités de son milieu et de son époque tout en prêtant sa parole à la cause nationale,

4 Benoît LaCroIx, RHAF 28.3 (1974), p. 415. 
Groulx a décuplé son rayonnement et assuré son influence à une époque où cette forme d'action était nécessaire. Il a ainsi réveillé un peuple de son engourdissement, redonné la fierté à ceux à qui leur existence collective semblait donner un complexe de honte et d'infériorité. D'autres considèrent qu'il aurait mieux fait de se confiner à la recherche et à l'enseignement académique. Groulx, faut-il le noter, qu'il ait écrit ou parlé, le faisait dans une langue d'une perfection remarquable. Supposons qu'il ait écrit des livres savants, que sa langue ait été inabordable, quelle influence aurait-il exercée sur une population qui n'était nullement préparée à le lire ou à l'entendre ? Et il y aurait une décennie déjà qu'on ne parlerait plus de Groulx, ni de son auvre. On ne le contredirait même pas, on ne le discuterait certainement pas. Et Groulx serait devenu emmuré dans son cabinet de travail ou dans sa bibliothèque. Ceux qui le jugent, oublient parfois que, par l'action et par la parole, Groulx a largement contribué à l'élaboration d'une pensée collective et au haussement du niveau de culture et à l'ouverture des esprits pour leur faire franchir quelques degrés de perfectionnement. La tranche historique de 1920 n'était certes pas au diapason de la tranche de 1960, pas plus au Québec qu'ailleurs.

Depuis quinze ou vingt ans, chez nous, et depuis 30 ou 40 ans en Europe, les historiens se sont davantage tournés vers des çntres d'intérêt précédemment négligés ou considérés moins importants. Ainsi à l'économique et au social ils ont finalement donné une place dans le champ de l'histoire. Mais, pourrait-on se demander, n'ont-ils pas eu tendance à leur tour à occuper tout le terrain, vu qu'ils le font ordinairement sans tenir aucun compte de la simultanéité de tout ce qui compose le reste de la vie, de cette vie que mène quotidiennement un peuple, comme un individu. À ce propos il paraît juste de signaler que Lionel Groulx fut notre seul et unique historien à englober dans sa vision toute l'histoire intégrale?

Enfin reprenons donc notre question du début. Lionel Groulx fut-il le grand homme qu'on nous a toujours dit? Pour répondre avec plus de certitude à une telle question, il faut, avons-nous dit, d'abord considérer cet homme en lui-même, c.a.d. dans ses œuvres, mais aussi ne pas oublier de tenir compte de son temps, de son milieu et de ses contemporains. Car, doit-on ajouter, Groulx a dû lutter pour défendre à la fois son intégrité et son indépendance de pensée et d'action. Il a lutté contre les hommes politiques et 
contre les autorités universitaires; il s'est défendu contre ses critiques et parfois il a dû garder ses distances vis-à-vis de ses propres disciplines quand ce ne fut pas face à ses admirateurs. À ce propos il convient de signaler quelques études d'égale valeur scientifique qui semblent répondre aux différents critères méthodologiques énoncés et appliquer par le détail les distinctions souhaitables, même si on peut en discuter les conclusions.

La première de ces études porte sur l'aspect économique de la société en Nouvelle-France. Dans son article de la RHAF, Jean Blain expose «Le cheminement historiographique dans la première moitié du $20^{\mathrm{c}}$ siècle ${ }^{5}$. Voici ce qu'il écrit à propos de Groulx :

Du reste, avec le recul des années, on peut voir que l'œuvre historique tout entière de Groulx vient confirmer ces tendances. L'Homme est foncièrement un historien de synthèse, passionné pour une histoire qui débouche moins sur la connaissance du passé, que sur les motifs que ce passé offre d'être fier aujourd'hui et confiant pour demain. Au total, il crée peu mais il restructure et présente admirablement bien les données acquises dans une langue d'une puissance évocatrice qui ne laisse pas encore aujourd'hui de séduire. Bref, un maître-diffuseur. (p. 15)

Et quelques pages plus loin, il précise davantage son jugement et son appréciation sur Groulx.

Jusqu'à la fin, le professeur de rhétorique devenu professeur d'université et historien de carrière resta un maître diffuseur. Cette vulgarisation - au sens premier du terme était sans doute nécessaire. Une étude approfondie pourrait certes montrer l'influence que la Nouvelle-France de Groulx (surtout l'influence de La naissance d'une race qui de 1918 à 1950 fut le seul ouvrage important de synthèse sur la période coloniale française) exerça sur les manuels d'histoire du Canada du niveau élémentaire, secondaire et collégial qui parurent de 1925 à 1960. (p. 23)

Et voici le jugement le plus grave :

Il est indéniable que cette emprise d'un homme, qui fut finalement beaucoup plus qu'un historien, a eu et continue

5 Jean BlaIN, «Économie et société en Nouvelle-France. Le cheminement historiographique dans la première moitié du $\mathrm{XX}^{\mathrm{e}}$ siècle ", in RHAF 26.1 (juin 1972), pp. 3-31. 
à avoir, dans des domaines autres que l'histoire, des effets importants. Il est certain aussi qu'elle est responsable de quelques vocations d'historiens éminents. Mais en même temps, on ne peut nier que l'ère de la diffusion que Groulx domine de toute sa personnalité s'accompagna d'un phénomène de stagnation et même de recul historiographique au niveau de l'interprétation officielle qui se voulait globale. (p. 23)

On a remarqué que Jean Blain constata «un phénomène de stagnation et même de recul historiographique » dans la pensée économique au temps de Groulx (1918-1950). Il ne démontre pas pourtant la responsabilité de Groulx mais dit qu'elle «s'accompagna ». Puis il passe ensuite aux «précurseurs sans audience» 6 .

En 1974, André J. Bélanger publiait L'Apolitisme des idéologies québécoises, ${ }^{7}$ couvrant «le grand tournant de 1934 à 1936 ». En gros son ouvrage converge vers Groulx et vers les média d'information susceptibles de diffuser sa pensée. Bélanger marque avec netteté la frontière entre le maître et ses disciples de La Nation et des Jeune-Canada. Ce qui lui permet de soutenir que Groulx n'était pas raciste et de rappeler opportunément qu'il n'a jamais prêché le retour à la terre. À cette étude de Bélanger on reproche l'emploi d'une grille théorique préétablie dite «théorie des fragments » qui ne devrait pas s'appliquer dans de tels cas ${ }^{8}$.

En troisième lieu vient une importante étude de Robert Comeau (RHAF, juin 1972, pp. 83-102) sur les «indépendantistes de $L a$ Nation et le séparatisme (1936-1938) $\gg{ }^{9}$. Comeau montre Groulx aux prises avec les empiètements de ses disciples. Ceux-ci se réclament de lui, mais leurs propos engendrent des ambiguïtés qui n'ont jamais cessé depuis de retomber sur leur Maître.

Face aux deux premiers écrits, d'autres chercheurs arrivent à d'autres conclusions sur la pensée économique et politique, sociale ou culturelle de Groulx. Dans un ouvrage d'apparence aussi modeste

6 Les «précurseurs » furent auteurs de monographies: FAUTEUX, Industrie au Canada (1929) ; SUlte et Tessier, Forges du St-Maurice (1920 + $1952)$; G. LangloIs, Population canadienne-française (1934) ou P.-G. RoY et ses inventaires d'archives publiés entre 1927 et 1941, concessions en fiefs, greffes de notaires, contrats de mariage, testaments ou encore des thèses jamais publiées.

7 PUL, 1979, 392 pp.

8 RHAF (juin 1975), pp. 101-103.

9 RHAF 26.1 (juin 1972), pp. 83-102. 
que son auteur, Jean-Louis Roy analyse le Maîtres chez nous de Groulx ou ses «dix années d'Action française (1917-1927)»10. Cette fois il s'agit non pas du maître et de disciples, mais du directeur et des collaborateurs ce qui n'est pas équivalent puisque avec lui ils mettent au point une doctrine avant de la diffuser dans la revue. D'une manière toute factuelle, J.L. Roy montre que leurs recommandations étaient d'une clairvoyance, d'un avant-gardisme et d'un réalisme étonnants soit sur la vie agricole et urbaine, la vie économique, soit sur la vie politique ou même sur la vie culturelle. On sait qu'en 1963, F.A. Angers affirmait de cette revue et de son prolongement L'Action nationale que pendant 50 ans et à elles seules, elles ont tenu le rôle de ministère des affaires culturelles au Québec.

Toujours d'après Roy, ce n'est pas en 1963 mais bien en 1921 que le «Maître chez nous» fut proclamé et que l'État a été considéré comme l'instrument de notre libération (p. 69). Dès 1918, on y parle de droit ouvrier, d'association ouvrière, d'enseignement agricole et technique et autres priorités. Puis on suggère à l'État de se munir d'instruments propres à assurer son action : un ministère des ressources naturelles, un ministère du Commerce et de l'Industrie.

Quant à la pensée et à l'influence politique de Groulx on a deux conférences de J.L. Roy qui sont la substance d'un ouvrage à paraître en novembre sous le titre probable de Terre-Québec. Ce fut aussi le commentaire servi ici en cette même salle à la conférence de M. F.A. Angers qu'il la publia en mai 1978 dans L'Action nationale sur «l'action politique de Groulx». Que cette pensée sociale, économique ou politique au dire de P.E. Trudeau n'ait pas « réussi à prendre corps dans des institutions dynamiques et vivantes ${ }^{11}$ ne prouve pas contre Groulx et ses collaborateurs, mais plus probablement contre les hommes politiques de l'époque qui ont tardé à en saisir le bien-fondé et la portée réelle.

Pourtant si l'on regarde du côté du peuple, comme l'a fait J.L. Roy pour la période consécutive à la guerre (1945-1960) soit tout le règne de Duplessis, on a des surprises. Dans La Marche des Québécois ${ }^{12}$ J.L. Roy montre assez factuellement qu'en dépit

10 Leméac, 1968, 75 pp.

11 La Grève de l'amiante, 1956, p. 11.

12 Jean-Louis RoY, La Marche des Québécois. Le temps des ruptures (1945-1960), Leméac, 1976, 383pp. 
d'une apparente stabilité et stagnation, le mouvement contestataire s'est étendu, élargi et organisé. "Les Québécois s'organisent 》. En 1977, André J. Bélanger dans Rupture et constantes ${ }^{13}$ analyse les idéologies de cette même période et nous montre entre autres sujets Lionel Groulx face à Cité libre.

Tous ces ouvrages et combien d'autres réfèrent au nationalisme de Groulx soit comme à la source principale de son inspiration, soit comme à l'origine d'une fausse vision des choses. Qu'en est-il au juste? Pour répondre nous avons un ouvrage de Léon Dion sur Les nationalismes et la politique au Québec qui couvre tout le début du $20^{\mathrm{e}}$ siècle, mais selon des phases à dominante idéologique ${ }^{14}$. Cet ouvrage fort intéressant fera un jour l'objet d'une refonte où l'auteur distinguera entre le nationalisme canadien de Henri Bourassa et le nationalisme québécois de Groulx même s'il les englobe dans le nationalisme conservatiste, selon son appellation. Comme Léon Dion a constaté la résurgence de ce nationalisme groulxien depuis dix ans ${ }^{15}$ il rejoindra les conclusions de Jean-Louis Roy dans Terre-Québec, et permettra à tous d'en admettre le bienfondé économique, politique et culturel, on aura ainsi rejoint Groulx. Et nous voici parvenus à notre deuxième question.

\section{B - LIONEL GROULX FUT-IL LE GRAND MÉCHANT HOMME QU'ON VEUT PARFOIS NOUS FAIRE CROIRE?}

Le 31 mai dernier, vous avez tous pu entendre à la radio une brève entrevue consacrée à notre historien national. Pressé de questions, l'interviewé présenté comme «un critique très prolifique de notre société québécoise », nous a en sept minutes fait l'honneur d'un menu des grands jours. Cette émission, dont nous avons écouté à plusieurs reprises l'enregistrement, nous a assuré que les propos suivants ont bien été tenus. Voici le message qu'elle contenait:

Lionel Groulx avait une pensée dangereuse que ses amis s'appliquent présentement à camoufler... Pensée dangereuse parce que fasciste, carrément fasciste... Il avait l'obsession

13 André J. BélANGer, Ruptures et constantes. Quatre idéologies du Québec en éclatement, HMH, 1977, 219pp.

$14 \mathrm{HMH}, 1975,177 \mathrm{pp}$.

15 Le Devoir, 19 novembre 1977. 
des hommes forts - (et soit dit en passant, n'allez pas penser à Victor Delamarre) - il s'agit plutôt de Franco, de Salazar, de Mussolini et même de Hitler... Groulx avait en outre une pensée statique, une conception autoritaire, de la société et de la famille, en un mot son culte de l'autorité voisinait ou véhiculait des valeurs nettement conservatrices... il était antisémite, intransigeant et intolérant... Finalement son histoire mythologique est aujourd'hui contredite par des historiens scientifiques...

Dans ce témoignage, il y avait donc peu de place pour l'admiration. Demandons-nous quelle importance son auteur accordait à la vérité.

Quiconque est le moindrement familier avec la pensée et l'œuvre d'un Lionel Groulx a beaucoup de difficulté à y reconnaître son homme. On se contenterait volontiers de sourire à l'audition de ces paroles si ce témoignage et d'autres du même calibre ne se rencontraient souvent dans nos journaux, nos revues et sur les lèvres même de nos plus jeunes élèves. S'il était vrai que de tels propos montrent une tendance à se répéter, à se diffuser, nous devons certes prendre quelques instants pour en chercher l'origine et les motivations, à en scruter l'auteur. Sans doute il ne serait pas erroné de soutenir que l'origine et les motivations sont multiples. Déjà par exemple quand on entend dénoncer les historiens patriopétards ou les agriculturistes, les fascistes et les racistes, on sent surgir en nos esprits certaines filiations ou paternités.

Au départ la nécessité s'imposerait sans doute de préparer une grande fresque idéologique dans le genre de celle que dresse un Jean-Marie Domenach dans un livre récent dont une tranche a paru en août dans la revue Esprit ${ }^{16}$. Domenach, que nous n'avons pas besoin de présenter, examine la place de «Dieu dans l'histoire » et relève les tentatives faites par les régimes totalitaires pour supprimer jusqu'à la présence divine. Il dégage quelques règles de sagesse qui l'ont guidé dans son enquête et qui seront utiles dans la nôtre. Les voici :

On ne saurait faire l'histoire d'une idée sans référence aux aspirations qu'elle satisfait, aux adversaires qu'elle combat, à la situation où elle s'incarne... J'écris ceci en un temps où des maoïstes confessent qu'ils ont cru en des dieux de papier,

16 Esprit, juillet-août 1978, pp. 87-96. 
et où Simone de Beauvoir déclare que "le socialisme est un rêve "... Lorsque les "nouveaux philosophes" cherchent la racine dı totalitarisme contemporain, ils partent dans un voyage à travers les philosophies... L'histoire du totalitarisme n'est pas une histoire philosophique, c'est une histoire religieuse... S'ils avaient un tant soit peu pratiqué la critique du témoignage, ils n'auraient pas sombré dans un délire dont ils font endosser la responsabilité aux philosophes du XVIII ${ }^{\mathrm{e}}$ siècle... Et maintenant, du même mouvement qu'ils crurent, ils voudraient nous faire dé-croire... Un peu plus de raisonnement aurait empêché ceux qui dénigrent aujourd'hui la raison de verser dans les idolâtries du siècle...

Tout en traitant d'un sujet aussi différent du nôtre, Jean-Marie Domenach établit des principes qui peuvent nous servir à la fois de guide et de stimulant. Car il est bien évident qu'en attaquant Groulx, pourtant disparu depuis onze ans, on vise bien au-delà de ses écrits et de sa pensée. D'autres personnes sont attaquées pour avoir partagé ses tâches et son crédo. D'autres idéologies que son nationalisme et d'autres disciplines que l'histoire sont également concernées. Mais établir de telles filiations d'idées nécessiterait de longues recherches que seules peuvent accomplir des équipes et encore avec quels résultats? Faisons tout de même quelques tentatives de sondages sur le contenu.

Les aspects idéologiques du nationalisme de Groulx ont tenté plus d'un penseur, même du vivant de Groulx. Cependant il est un ouvrage qu'on cite parfois à l'égal d'une Bible en ce domaine et qu'on utilise aussi sans même le nommer. Une première lecture de cet ouvrage fait plus que nous laisser déconcerté, elle nous le révèle finalement comme une source plus que probable des propos cités plus haut, avec ou sans déformation. Cet ouvrage s'intitule Le Nationalisme de Lionel Groulx ${ }^{17}$. Signé en août 1969 par Jean-Pierre Gaboury, et paru sur le marché en mai 1970, il témoigne incontestablement d'un effort sérieux et d'une somme considérable de recherches et de lectures. Disons tout de suite que sa partie la plus valable est la Bibliographie de Lionel Groulx qui couvre les 25 dernières pages. Comme elle n'entre pas directement dans l'étude des aspects idéologiques annoncée, elle constitue une sorte d'appendice, une annexe.

17 Jean-Pierre Gaboury, Le Nationalisme de Lionel Groulx. Aspects idéologiques, Université d'Ottawa, 1970, 226pp. 
Quand on lit ce livre, on se défend mal d'un certain malaise dont on ne discerne pas la nature à première vue. Un examen plus poussé montre que tout en étant consacré aux «aspects idéologiques », cet ouvrage comporte ses principales carences précisément à ce niveau idéologique. Les unes sont pourtant plus graves que les autres, même s'il est évident qu'elles ont échappé à leur auteur autant qu'au patron et aux lecteurs de la thèse.

L'auteur est peut-être d'origine franco-ontarienne. Il avait poursuivi de longues recherches aux archives de Lionel Groulx qu'il semble même avoir interviewé de son vivant. Puis il alla en France poursuivre des études en sciences politiques à la Faculté de Droit et de sciences économiques de l'université de Paris. Sa thèse y fut rédigée sous la direction du professeur Jean-Jacques Chevallier et elle constate la complexité du sujet dès son début. Mais, peut-on se demander, lui et les autres lecteurs de la thèse étaient-ils suffisamment en garde pour songer à vérifier les citations, la langue, la philosophie et même les idéologies engagées dans ce travail ?

De façon inexplicable, tout ce bon monde n'a accordé aucune importance à la dimension chronologique qui aurait pu donner à cette recherche son véritable sens historique, pourtant indispensable à un examen critique de ce genre. Une première tranche de l'étude, le chapitreV consacré à la politique, fut même publiée dans $L^{\prime}$ Action nationale de juin 1968 .

Deux lectures consécutives du livre de Gaboury, faites la plume à la main, nous ont personnellement conduit l'été dernier aux constatations que voici. En tout premier lieu, l'auteur ne maîtrise pas très bien toute la philosophie sous-jacente à son livre. Ce qui a pour conséquence de ne pas localiser très bien l'auteur dans le tableau idéologique et du même coup par rapport à son sujet d'étude. Troisième effet qui découle de là, vu la rigueur de certains jugements de valeur qu'il énonce, l'auteur offre de lui-même à son lecteur une image méprisante et hautaine ${ }^{18}$. Ce qui n'est pas vrai dans la réalité. De plus il lui arrive aussi de raisonner plutôt avec des mots qu'avec des concepts. Ce qui le conduit à des conclusions qui vont parfois au-delà de ses démonstrations.

Pour ce qui est de la langue, elle trahit son auteur autant au niveau de la pensée qu'au niveau de l'expression. Et à cause de la

18 Cf. p. 95, p. 102. 
discordance qu'on remarque parfois entre le texte et certaines références ${ }^{19}$, on est légèrement induit en erreur. Ce qui est excusable au plan bibliographique ne l'est nullement dans le domaine des idéologies. Et finalement qu'il cite du Maritain ou du Maurras comme s'il s'agissait du Groulx, peut avoir des conséquences.

Touchant la langue ou l'expression, voici un exemple où Gaboury écrit une première fois que Groulx a «créé »l'histoire de la Nouvelle-France. Parler de création en histoire peut être une expression qui passe une fois, mais quand elle se maintient pendant une page ou deux elle produit la surprise ${ }^{20}$. Elle laisse finalement l'impression que l'histoire est une œuvre de création, d'invention, d'imagination. Ảlors que même romantique l'histoire n'est jamais un roman.

Plusieurs citations faites par Gaboury, pour être demeurées trop longtemps sur fiches avant de prendre place dans le corps du texte au moment de sa composition, ont fini par perdre jusqu'à leur lien naturel avec le contexte d'où elles sont tirées ${ }^{21}$. Ainsi s'explique qu'elles font dire à l'auteur cité le contraire de sa pensée. $\mathrm{Au}$ total, pourrait-on conclure, cet ouvrage qui a valu un doctorat pour l'effort qu'il représente et que nous ne voulons nullement contester, aurait certes gagné à être revu en profondeur ou même à être refondu avant d'aller aux presses et par là aux lecteurs, vu l'importance des engagements qu'il entraîne avec lui.

Les critiques écrites ou orales faites sur cet ouvrage ont été plutôt modérées. Elles sont d'accord sur deux points : la difficulté et la complexité du sujet et la non culpabilité de l'auteur. Mais ce qui paraît plus grave à nos yeux découle des deux lectures faites cet été, lectures entreprises en toute disponibilité d'esprit sans intention préalable d'incriminer l'auteur ni son œuvre. Ces lectures nous ont permis de relever, parfois nettement formulées, tantôt simplement insinuées ou esquissées, les graves accusations contre Lionel Groulx que vous avez entendues il y a un instant ou que vous avez pu lire dans Le Devoir des 18, 19, 20 et 23 mai ou encore dans des revues littéraires ou philosophiques à tendance socialisante. Les jugements et conclusions du livre de Gaboury et de tous ces écrits mentionnés, sont parfois très loin et de la pensée et des écrits de Groulx comme

19 Pp. 102-103, 108, 109, 112, tout le chap. IV, L'histoire.

20 Voir chap. IV, L'histoire, pp. 92, 99, 103, 121.

21 RHAF 24 (sept. 1970), pp. 287-290. 
elles sont éloignées des constatations qui ressortent d'un inventaire sérieux des écrits de Groulx. Deux critiques auxquelles nous ferons appel furent publiées. L'une dès septembre 1970 dans RHAF par Richard Arès, l'autre par Ramsay Cook en novembre de la même année dans Histoire sociale de l'université d'Ottawa.

Quand Pierre Vallières reproche à Lionel Groulx d'avoir une pensée statique, il rend tout simplement l'impression qui se dégage du livre de Gaboury. C'est même un des principaux reproches formulés par Ramsay Cook. En ne faisant qu'un seul bloc des 65 ans d'activité de Groulx, sans aucune référence ni au temps, ni au milieu, ni au contemporains de Groulx, Gaboury le réduit au penseur en chambre, au philosophe dans une tour d'ivoire, au vivant en marge de son temps en plus de lui prêter une pensée statique et monolithique. Ce qui est contraire à la vérité. Et ce monolithisme se fait sentir même dans l'expression lorsque $\mathrm{Ga}$ boury confirme une pensée de 1965 par un texte de 1917. Ne pas situer Groulx dans son époque, ni dans son milieu, c'était le priver et nous priver à la source des réactions et des prises de position qui ont été les siennes et du même coup les rendre incompréhensibles. Ramsay Cook note avec justesse : " c'est ce sens de la dynamique, ou ce sens de l'évolution qui manque dans la méthode d'approche de Gaboury ». Et au lieu de mesurer Groulx à son pays et à ses contemporains, on ne peut que le juger à l'aune des Européens et au vecteur des siècles passés. Finalement, pour reprendre une remarque de Ramsay Cook, c'est Gaboury lui-même qui devient victime à l'égal de Groulx de son propre procédé. Et Cook signale que la pensée de Groulx a évolué autant en économique qu'en politique. Et nous ajouterions que son évolution s'est faite même dans sa conception de l'histoire et dans ses procédés méthodologiques.

Il est aujourd'hui bien établi que le nationalisme de Groulx fut différent du nationalisme de Henri Bourassa. Pour celui-ci le nationalisme n'avait d'autre fondement que politique et prenait naissance face à l'impérialisme britannique, tandis que pour Groulx le nationalisme s'enracinait à la fois dans l'origine ethnique, dans la culture et dans la langue, dans la vie sociale et religieuse. Toutes choses qui ont constamment justifié les attitudes de Groulx face à la Confédération et face au BNAA (AANB). Si on le préfère, l'attitude de Groulx conditionnait ses rapports avec l'aspect politique et avec l'aspect constitutionnel de l'histoire du Canada. Autrement 
dit, Groulx avait découvert que la constitution de 1867 demeurée pendant cent ans ce qu'elle était dès son début, avait dans son application enfreint ou contredit l'esprit autant que la lettre du BNAA.

Venons-en ensuite au racisme de Groulx qui peut, si l'on veut, englober son antisémitisme et sa xénophobie anti-anglaise. Reconnaissons que certaines ambiguïtés de vocabulaire avaient généralement cours à l'époque où il écrivait La naissance d'une race ou L'Appel de la Race. Cependant quand les circonstances eurent évolué, Groulx s'expliqua, puis eut recours à des termes comme «le petit peuple » ou «la nation » qui gardaient à sa pensée tout son sens vrai. Bien loin d'être xénophobe ou raciste, Groulx citait à ses co-nationaux l'exemple des Juifs et des Anglais en guise d'invitation à imiter leur comportement, leur solidarité, leur fierté du passé et de la culture pour ensuite demander ou prendre comme leurs libertés politiques, culturelles ou linguistiques. Autrement dit Groulx réclamait pour les siens une place au soleil, une part de l'héritage d'Adam. Les textes ne manquent pas non plus où il affirme que son préjugé favorable aux Canadiens français ne signifie nullement une opposition aux autres nations du Canada ou du Québec.

Revenons aux propos de notre apprenti politicologue où il révèle son manque de maîtrise dans ses propres concepts. Prenons l'exemple de son analyse du «chef» que cherchait Groulx. Sans même faire de nuance ou de précisions utiles, il affirme que désirer un chef c'est aller contre l'esprit démocratique (p. 150). Pourtant un texte ignoré de Gaboury et cité par Ramsay Cook montre que Groulx avait du chef une conception qu'on appellerait aujourd'hui charismatique. Sans doute la théorie et la pratique de la démocratie ont largement évolué depuis 40 ans, tant chez nous que dans le reste du monde. Mais à une époque comme la nôtre, où l'on fait constamment appel au leadership, il ne semble pas possible de confondre dictateur et chef, même si celui-ci est nationaliste. En outre, on sait très bien que dans les années '30, ni Dollfuss, ni Salazar, ni Pétain, ni Franco, ni Mussolini, ni même Hitler n'étaient perçus comme dictateurs. Ni les Autrichiens, ni les Portugais, ni les Italiens, ni les Allemands n'avaient encore vécu les années 1940 ou 1950. Seuls les prophètes après coup peuvent commettre de si graves erreurs et montrer une telle ignorance de l'histoire et un tel oubli de la dimension chronologique des idées. Dans la galerie des noms que Gaboury rattache au nationalisme de Groulx, on peut 
relever peut-être une couple de mentions dans les écrits de Groulx. Et encore le procédé n'était pas de son invention, ni son monopole. Et l'on remarquera qu'il ne les citait pas comme dictateurs, mais plutôt comme chefs charismatiques ou leaders. Quant à en faire une "obsession chez Groulx», c'est nettement abuser des mots, alors qu'il serait préférable d'ajuster son information et son vocabulaire avant de parler. Quant au nationalisme et à l'antisémitisme, voyons ce qu'en a écrit Jean-Marie Domenach.

On ne peut pas plus assimiler Nietzsche à Hegel comme " antisémite " qu'on ne saurait, au $\mathrm{XX}^{\mathrm{e}}$ siècle, bloquer sous le nom de nationalisme Maurras et Frantz Fanon, de Gaulle et Pinochet, Hitler et Boumedienne. L'antisémitisme s'est nourri de tout, comme le nationalisme aujourd'hui se nourrit de tout, y compris du judaïsme... Ce qui importe, c'est de chercher à quelle exigence souterraine répondent ces nationalismes, car ils ont, très profondément, une racine commune, de même que les antisémitismes en avaient une que Hegel a mis au jour dans L'esprit du christianisme et son destin.

En poursuivant nos lectures, nous avons cru, l'espace d'un matin, qu'il avait un rapport direct, de cause à effet, entre l'ouvrage de Gaboury et celui de Suzan Mann Trofimenkoff ${ }^{22}$. Ce qui donne cette impression est le rôle de Ramsay Cook, critique de Gaboury, qui de Toronto a dirigé son étudiante à la fois vers la pensée de Lionel Groulx et vers l'université Laval pour y étudier le nationalisme canadien-français, le rôle de L'Action française et la teneur de L'Appel de la race. Même un examen strict de la chronologie ne parvient pas à dissiper complètement cette impression pour l'instant. Dans sa préface Suzan Trofimenkoff établit certains éléments de sa veine.

Les éléments de chance qui accompagnent la préparation d'un livre sont parfois extraordinaires. Si je n'avais pas rencontré le professeur Ramsay Cook à l'université de Toronto, je n'aurais jamais enseigné l'histoire du Canada ni écrit à propos du nationalisme canadien-français. Si j'étais demeurée en Ontario pour mes études doctorales, ce livre aurait été très différent. Le bénéfice, je crois, de l'année passée sous l'enthousiaste et encourageante direction du professeur Pierre Savard, et les trois ans d'enseignement que j'ai fait à l'université de

22 Susan Mann Trofimenkoff, Action française. French Canadian Nationalism in the Twenties, U. of Toronto Press, 1975, 157pp. 
Montréal m'ont permis d'apprendre de la bouche de mes étudiants davantage sur le nationalisme que je leur ai enseigné sur l'histoire du Canada.

À ces conditions préparatoires se sont ajoutées chez Madame Trofimenkoff d'autres conditions de maturation. En 1970, l'année même où elle termine son doctorat, elle publie une étude sur le nationalisme de Bourassa et sur celui de Groulx dans la décennie de $1920^{23}$. Tout en poursuivant son enseignement à Montréal, elle prépare la publication d'une remarquable anthologie de Groulx ${ }^{24}$. Benoit Lacroix, lui-même auteur d'une anthologie, souligne l'attention qui a été portée à la traduction des textes. C'est que l'auteur était bien consciente que respecter la langue c'était respecter la pensée. Puis finalement Suzan Trofimenkoff publie en 1975 une partie de sa thèse de doctorat Action française. French Canadian Nationalism in the Twenties. (156 pp.)

Nous ne voulons pas pour le moment porter jugement sur les écrits de Madame Trofimenkoff parce que n'avons pas complété lecture et observations. Mais déjà nous avons été frappé par le soin qu'elle montre non seulement à la langue et à la pensée, mais à la chronologie et aux brèves périodes qu'elle accorde à son étude. À l'occasion de cette anthologie, préparée par Trofimenkoff, Benoit Lacroix fait remarquer qu'en

Relisant tout dernièrement ces textes bien courts pourtant de Lionel Groulx, nous ne pouvions pas ne pas l'imaginer tout de suite en croisade contre tout ce qui pourrait réduire, simplifier le fait canadien-français en Amérique du Nord. Ô son Amérique française! Face à ce passé qu'il aimait trop pour ne pas l'embellir, nous nous demandions ce qu'il dirait aujourd'hui de toute cette mythologie québécoise à propos de notre mentalité d'assiégés, notre soi-disant et bien triste résignation chrétienne devant le conquérant, sur la passivité paysanne, sur ce "pauvre clergé mal instruit " qui aurait prêché collaboration et soumission à l'ennemi, sans oublier le joual et la redécouverte nostalgique de ces bons indiens tant exploités par la bourgeoisie! Comment aurait-il accepté cette dialectique de la haine des uns pour mieux aimer les autres?

23 Robertson (TrofimenkofF), "Variations on a Nationalist Theme : Henri Bourassa and Abbé Groulx in the 1920's », CHA, 1970, pp. 109-119.

24 Trofimenkoff, Abbé Groulx: Variations on a Nationalist Theme, Copp. Clark, 1973, 256pp. 
Comment aurait-il pu résister à 10 heures du matin à l'idée d'un autre article dans Le Devoir contre ceux qui tendent à réduire l'avenir du Québec à des luttes de classe, à un combat salarial, à un seul parti politique ou même à la guerre linguistique? Il aurait surtout eu peur des raccourcis idéologiques d'un certain néo-nationalisme importé on sait bien d'où et qui n'a rien ou fort peu à voir jusque dans ses slogans avec lui et son œuvre.

Lui qui fut si longtemps et comme par instinct un vif partisan d'une religion nationale protectrice des droits et de la langue, comment aurait-il accepté aujourd'hui l'évolution attendue des mentalités sans références explicites au spirituel ? Il tenait trop à la qualité d'âme des siens pour ne pas souffrir, déjà en 1960, et parfois mal, de ce qu'il pressentait sans trop vouloir y croire. Ou peut-être aurait-il à la longue et devant le dynamisme de la culture québécoise de ces dernières années aimé ces temps de la purification nécessaire, et jusque dans les blasphèmes légèrement cruels des siens entrevu une manière bien québécoise encore d'exorciser un passé abusif ? 25

\section{CONCLUSION}

En conclusion, disons que malgré son admiration et son enthousiasme, Benoit Lacroix est demeuré conscient des difficultés que rencontrera de plus en plus l'interprète de la pensée de Groulx. En voici la preuve :

De plus en plus, écrit-il, à cause des changements inévitables de mentalités et à cause des nouvelles techniques d'écriture et de critique, l'interprétation de Groulx posera des problèmes méthodologiques. Ainsi, en 1974, faudrait-il l'étudier par réfractions, au nom du. signifiant plutôt que du signifié, revenir aux archétypes plutôt qu'aux conclusions, le surprendre dans ses réflexes plutôt que de l'attendre à la fin d'un paragraphe, en appeler au sacré plutôt qu'à sa seule foi catholique, au besoin le séculariser pour mieux l'accepter ensuite dans sa totalité ? Comment faire la part en lui, par exemple, de la religion officielle des sermons, des retraites, et de plusieurs discours de circonstance, quand nous apprenons par ailleurs en lisant ses Mémoires qu'il fut aussi et même beaucoup le croyant à la foi davantage naïve et populaire faite

25 RHAF, juin 1974, pp. 415-420. 
de dévotions et de croyances qu'on retrouve plutôt chez les paysans que chez les intellectuels.

Le temps et l'espace sont des contraintes qui ont limité cette analyse à une esquisse. Mais chacun pourra toujours compléter à l'aide des lectures indiquées. Pour le moment toutefois cette analyse justifie pleinement les conclusions suivantes :

1- La pensée fasciste n'est certes pas du côté indiqué dans le témoignage cité au début.

2- On peut en dire autant de «la pensée dangereuse» et du camouflage.

3- Les historiens scientifiques corrigent et complètent souvent Groulx, mais ils contredisent surtout ses détracteurs.

4- Quant à l'intransigeance et à l'intolérance, au mythe et à la mystique qui les sous-tend, elle est évidemment ailleurs que chez Groulx.

Et nous cédons le mot de la fin à Jean-Marie Domenach qui dit :

«J'écris ceci en un temps où des maoistes confessent qu'ils ont cru en des dieux de papier et où Simone de Beauvoir déclare que «le socialisme est un rêve».»

Georges-Émile GIGUÈrE 